Research Article

Ali Gholami Rudi*

\title{
Approximate Discontinuous Trajectory Hotspots
}

https://doi.org/10.1515/comp-2020-0176

Received Aug 13, 2019; accepted May 06, 2020

\begin{abstract}
A hotspot is an axis-aligned square of fixed side length $s$, where the amount of time a moving entity spends within it is maximised. An exact hotspot of a polygonal trajectory with $n$ edges can be found with time complexity $O\left(n^{2}\right)$. We define a $c$-approximate hotspot as an axisaligned square of side length $c s$, in which the presence duration of the entity is no less than that of an exact hotspot. In this paper we present an algorithm to find a $(1+\epsilon)$ approximate hotspot of a polygonal trajectory with time complexity $O\left(\frac{n \phi}{\epsilon} \log \frac{n \phi}{\epsilon}\right)$, where $\phi$ is the ratio of average trajectory edge length to $s$.
\end{abstract}

Keywords: Computational geometry, geometric algorithms, trajectory analysis, trajectory hotspots

2010 Mathematics Subject Classification: 68U05

\section{Introduction}

Many objects on earth move and huge collections of trajectory data have been collected by tracking these objects with technologies such as GPS devices. In the analysis of these trajectories, many interesting geometric problems arise, such as simplification [1], segmentation [2], grouping [3], classification [4], and finding interesting regions, such as those in which objects spend a significant amount of time [5-9].

Few results have been published which present exact geometric algorithms for the identification of regions that are frequently visited. Hereafter, we shall call such regions hotspots. It should be noted, however, that several heuristic algorithms have been published, such as [7]. The movement of an entity (its trajectory) is commonly represented as a polygonal curve. A set of vertices show the location of the entity at specific points in time, and line segments (edges) connect contiguous vertices. For multiple entities, Benkert et al. [5] defined a hotspot as an axis-

^Corresponding Author: Ali Gholami Rudi: Department of Electrical and Computer Engineering, Babol Noshirvani University of Technology, Babol, Mazandaran, Iran; Email: gholamirudi@nit.ac.ir aligned square, which is visited by the maximum number of distinct entities. They presented an $O(n \log n)$ time sweep-line algorithm, where $n$ is the number of trajectory vertices, when only the inclusion of a trajectory vertex is considered a visit. For the case where the inclusion of any portion of a trajectory edge is a visit, they presented an $O\left(n^{2}\right)$ algorithm, which subdivides the plane to find hotspots. They showed in both cases that their algorithm is optimal.

In a more recent paper, Gudmundsson et al. [6] examined different definitions of trajectory hotspots, in which the duration of the entity's presence in a region is significant. In this paper, we focus on one of their definitions, which is given as follows: a hotspot is an axis-aligned square of some pre-specified side length, in which the entity (or entities) spends the maximum possible duration, and the presence of the entity in the region can be discontinuous. For this problem and for a trajectory with $n$ edges, they presented an exact $O\left(n^{2}\right)$ algorithm. It subdivides the plane based on the breakpoints of the function that maps the location of a square of the specified side length to presence duration of the entities in that square. Using kinetic data structures, Rudi [9] presented a faster, $O\left(n \log ^{3} n\right)$ time 2-approximation algorithm, for identifying such regions when the edges of trajectories are axisaligned. In some applications, the entities are allowed to leave the region, but the duration of the entity's absence from the region should be bounded. Rudi [8] presented an $O(n \log n)$ time $(1+\epsilon)$-approximation algorithm to identify such regions.

When $s$ is the side length of exact hotspots, a $c$ approximate hotspot, where $c>1$, is an axis-aligned square of side length $c s$, in which the duration of the entity's presence is no less than that of an exact hotspot. In this paper we present an algorithm to find $(1+\epsilon)$ approximate hotspots of a trajectory in the plane. The algorithm first subdivides each edge into small segments and then finds the square that contains the maximum number of such segments. The time complexity of the algorithm, which shall be presented in the rest of this paper, is

$$
O\left(\frac{n \phi}{\epsilon} \log \frac{n \phi}{\epsilon}\right),
$$

where $\phi$ is the ratio of average length of trajectory edges to $s$. We then use this algorithm to find a duration- 
approximate hotspot of a trajectory $T$ with approximation ratio $1 / 4$, i.e. a square of side length $s$, in which the entity is present at least $1 / 4$ of the time it is present in the exact hotspot.

This paper is organised as follows. The algorithm and its analysis are presented in Section 3, after introducing the notation and defining some of the concepts discussed in this paper in Section 2. Finally, we conclude this paper in Section 4.

\section{Preliminaries}

A trajectory describes the movement of an entity and is represented as a sequence of vertices in the plane with timestamps that specify the location of the entity at different points in time. The entity is assumed to move from one vertex to the next in a straight line and with constant speed. This is formalised in Definition 2.1.

Definition 2.1. A trajectory is a sequence of vertices that represent the location of a moving entity at different point in time. The time at which the entity is observed at vertex $v$ is denoted as $t(v)$. Contiguous vertices of the sequence are connected by an edge, i.e. a segment that represent the movement of the entity between its endpoints.

We define the weight of a square or an edge as follows (Definition 2.2).

Definition 2.2. The weight of a square $r$ with respect to $a$ trajectory $T$, denoted as $w(r)$ is the total duration of time in which the entity spends inside the square. Also, $w(u)$ for any sub-trajectory (or edge) $u$ of $T$ indicates the amount of time the entity spent moving through $u$ (the difference between the timestamps of its endpoints).

Let $\ell_{u}$ denote the length of edge $u$ and let $I_{r}(u)$ denote the length of the portion of edge $u$ that is inside square $r$. Then, we have:

$$
w(r)=\sum_{u \in T} w(u) \cdot \frac{I_{r}(u)}{\ell_{u}} .
$$

The input to the problem studied in this paper is a trajectory $T$ and the value of $s$, where $s$ is a real. The goal is to find a hotspot of $T$ of side length $s$ (Definition 2.3). Unless explicitly mentioned otherwise, every square discussed in this paper is axis-aligned and has side length $s$.

Definition 2.3. A hotspot of trajectory $T$ in $\mathbb{R}^{2}$ is a placement of a square of side length $s$ in the plane with maximum weight (the amount of time the entity spends in that square is maximised).

A hotspot of a trajectory with $n$ edges can be found with time complexity $O\left(n^{2}\right)$ [6]. Our goal in this paper is finding approximate hotspots of a trajectory more efficiently (Definition 2.4).

Definition 2.4. A c-size-approximate (or c-approximate for brevity) hotspot is a square whose weight is at least the weight of an exact hotspot and its side length is c times s.

In Definition 2.4, hotspots are enlarged. We may alternatively define approximate hotspots by keeping their size fixed, but allowing their weights to be smaller than that of an exact hotspot (Definition 2.5).

Definition 2.5. A c-duration-approximate hotspot is a square of side length $s$, whose weight is at least c times the weight of an exact hotspot.

\section{The Approximation Algorithm}

Let $T$ be a trajectory in $\mathbb{R}^{2}$ and $\epsilon$ some constant such that $\epsilon>0$. In this section we present an algorithm to find a ( $1+$ $\epsilon$ )-approximate hotspot and use it to find a 1/4-duration approximate hotspot.

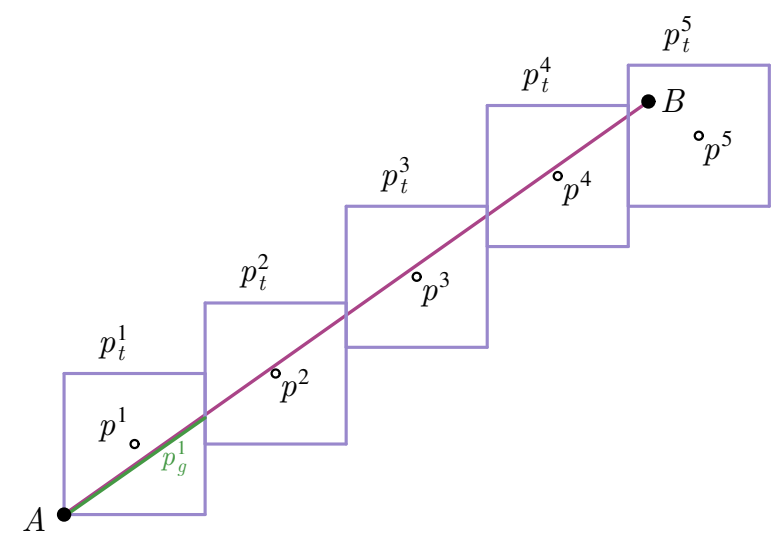

Figure 1: Tiling an edge $A B$ and adding a point at the centre of each tile; $p^{i}$ is the centre of the $i$ th tile $\left(p_{t}^{i}\right)$.

We first subdivide each edge of the trajectory into segments of length at most $\epsilon s / \sqrt{2}$; we do so by covering each edge by non-overlapping axis-aligned squares of side length $\epsilon s / 2$ (referred to as tiles hereafter). We can minimise the number of resulting tiles using Algorithm 3.1. Note that it does not affect the time complexity of the algo- 
rithms that are presented later asymptotically if the number of resulting segments is not the minimum though.

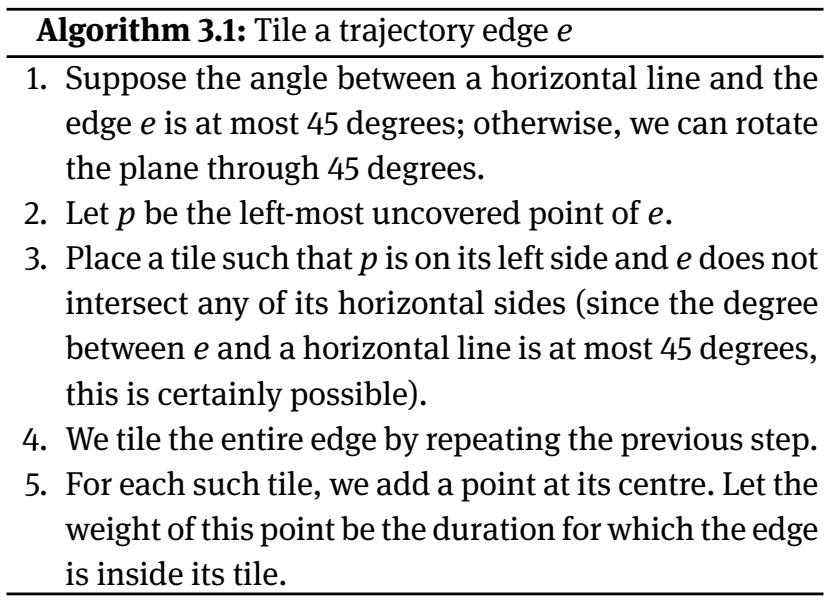

The number of resulting tiles for an edge of length $d$ is at most $\left\lceil\frac{2 d}{\epsilon s}\right\rceil$. For an edge $u$, let $P_{u}$ denote the set of points added after tiling $u$. For every resulting point $p$, we use $p_{t}$ to denote its corresponding tile and $p_{g}$ to denote the corresponding segment (Figure 1).

Definition 3.1. The point-weight of a square $r$ with respect to a trajectory $T$, denoted as $w^{\prime}(r)$, is the total weight of points inside $r$ after tiling all edges of T. Also, for point $p$, $w^{\prime}(p)$ denotes the weight of point $p$.

Therefore, after tiling an edge $u$, we have for a point $p \in P_{u}$

$$
w^{\prime}(p)=w(u) \cdot \frac{I_{p_{t}}(u)}{\ell_{u}},
$$

and for a square $r$ we have

$$
w^{\prime}(r)=\sum_{u \in T}\left(\sum_{p \in P_{u} \text { and } p \in r} w^{\prime}(p)\right) .
$$

Note that tiles of different edges may overlap.

Lemma 3.2. Let $r$ be a square of side length $s$ and let $r^{\prime}$ be a square of side length $s+\epsilon s / 2$, with the same centre of gravity. After tiling a trajectory $T$, we have $w(r) \leq w^{\prime}\left(r^{\prime}\right)$.

Proof. Every point in the sub-trajectory inside $r$ belongs to some segment $p_{g}$ corresponding to the tile $p_{t}$ and the point $p$. Let $P$ be the set of all points whose segments are intersected by $r$. Since $r$ contains all or some part of any segment corresponding to these points,

$$
w(r) \leq \sum_{p \in P} w(p) .
$$

Since $r^{\prime}$ is $\epsilon s / 4$ longer than $r$ on each side, when $r$ intersects $p_{t}$, the centre $p$ of $p_{t}$ is contained in $r^{\prime}$ (see Figure 2). Therefore,

$$
\sum_{p \in P} w(p) \leq w^{\prime}\left(r^{\prime}\right),
$$

which implies that

$$
w(r) \leq w^{\prime}\left(r^{\prime}\right) .
$$

Lemma 3.3. Let $r^{\prime}$ be a square of side length $s+\epsilon s / 2$ and let $r^{\prime \prime}$ be a square of side length $s+\epsilon s$, with the same centre of gravity. After tiling a trajectory $T$, we have $w^{\prime}\left(r^{\prime}\right) \leq w\left(r^{\prime \prime}\right)$.

Proof. Let $P$ denote the set of points inside $r^{\prime}$; the pointweight of the square $r^{\prime}$ is the sum of the weights of the points in $P$. Suppose $p$ is a member of $P$. Since $p$ is the centre of $p_{t}$ and $p$ is inside $r^{\prime}$, the whole of $p_{t}$ is contained in $r^{\prime \prime}$ because $r^{\prime \prime}$ is $\epsilon s / 4$ longer than $r^{\prime}$ on each side (demonstrated in Figure 2). This implies that the whole of $p_{g}$ is inside $r^{\prime \prime}$. Therefore,

$$
\sum_{p \in P} w^{\prime}(p)=\sum_{p \in P} w\left(p_{g}\right) \leq w\left(r^{\prime \prime}\right),
$$

as required.

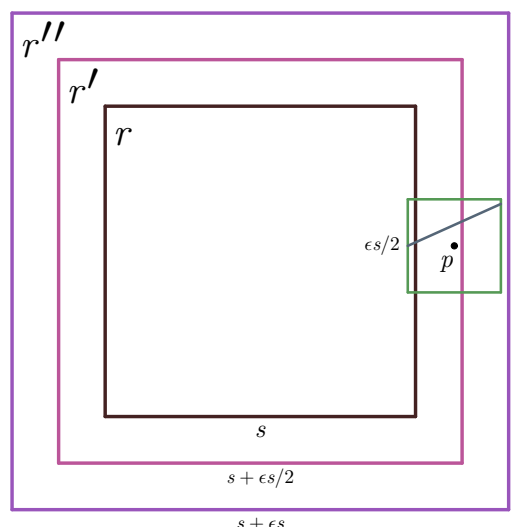

Figure 2: If $p_{g}$ is (maybe partially) in $r$, the centre of tile $p_{t}$ is contained in $r^{\prime}$ (Lemma 3.2), and if the centre of a tile is in $r^{\prime}$, it is completely inside $r^{\prime \prime}$ (Lemma 3.3).

Remark 3.4. In Theorem 3.5, we use a data structure for storing $m$ numbers that supports obtaining their maximum in $O(1)$, and adding a value to any continuous interval of the numbers in $O(\log m)$. This can be implemented using a balanced binary tree. The data structure is well-known (especially in computer science programming contests); we shall briefly explain its implementation here. Let v.val be 
the value stored at node $v$. Input numbers are stored at the leaves. The updated value of a leaf $u$ is the sum of its value and $v . v a l$ for every ancestor $v$ of $u$. Therefore, for instance, increasing the value stored at the root of the tree by $c$ is equivalent to increasing the value stored at every leaf by $c$.

For every internal node $v$, we also maintain v.max, which points a leaf with the maximum value among those under $v$. The value of $v$. max for every node $v$ of the tree can be initialized recursively in $O(m)$. Note that changing v.val does not change v.max because the relative values of the leaves under $v$ do not change (all of them are changed by the same amount) but it may change u.max for some of the ancestors of $v$.

Suppose we want to increase the value of leaves $i$ through $j$. We can update v.val for every such leaf and then update v.max for any of their ancestors. The time complexity of that would be $O(m)$. Instead, we can start from the root and perform the following steps. For each such node v:

1. If the leaves under $v$ do not intersect the update range $(i, j)$, there is nothing to be done for $v$.

2. If the leaves under $v$ are all inside $(i, j)$, we can simply increase v.val (that would increase the value of every such leaf).

3. If only some of the leaves under $v$ are inside $(i, j)$, we recursively check the children of $v$.

For the third case, we also update the value of v.max after the recursive call. The time complexity of this algorithm is $O(\log m)$, because it can be shown (using the same proof as in range trees [10]) that at every depth at most two nodes are checked.

Theorem 3.5. Suppose we are given a trajectory $T$ in $\mathbb{R}^{2}$ and the value of s. Then, after tiling, a square of side length $s+\epsilon s / 2$ and with the maximum point-weight can be found with time complexity $O(m \log m)$, where $m$ is the number of points.

Proof. To find a square with the maximum weight, it suffices to search among the squares that have a point on each of their lower and left sides (any square with the maximum weight can be moved up and right without changing its point-weight, until their lower and left sides meet a point).

Let $\sigma$ be the sequence of points in $P$, ordered by their $y$-coordinate. We sweep the plane horizontally using two parallel sweep lines with distance $s+\epsilon s / 2$ as follows. During the sweep line algorithm, we maintain the point-weight of $m$ squares in the data structure $W$, such that the $i$-th number in $W$ denotes the point-weight of the square whose lower side has the same height as the $i$-th point, and its left and right sides are on the sweep lines; we use $r_{i}$ to refer to this square.

In the sweep line algorithm, we process the following events:

- When a point $p$ intersects the left sweep line.

- When a point $p$ intersects the right sweep line.

We process an event for the point $p$ as follows.

1. Let $p$ be the $i$-th item of $\sigma$ and let $j$ be the index of the lowest point in $\sigma$ such that the difference between the height of $p$ and the $j$-th point of $\sigma$ is at most $s+\epsilon s / 2$; the value of $j$ can be found using a binary search on $\sigma$.

2. When $p$ meets the right sweep line, we increase the weight of every square $r_{k}$ such that $j \leq k \leq i$ by $w^{\prime}(p)$, because every such square contains $p$. Similarly, when $p$ meets the left sweep line, we decrease the weight of every square $r_{k}$ such that $j \leq k \leq i$ by $w^{\prime}(p)$.

During the sweep line algorithm, we record the square with the maximum weight in $W$. At the end of the algorithm, it denotes a square with the maximum point-weight among all squares with a vertex on their lower and left sides.

The time complexity of handling each event is $O(\log m)$. Therefore, the time complexity of sorting $m$ points by their $y$-coordinate, and handling $m$ events is $O(m \log m)$.

In Theorem 3.6, we prove the correctness of our main algorithm, i.e. Algorithm 3.2.

\begin{tabular}{l} 
Algorithm 3.2: Find a $(1+\epsilon)$-approximate \\
hotspot of trajectory $T$ \\
\hline 1. Tile the edges of $T$, as described in Algorithm 3.1. \\
2. Use the algorithm mentioned in Theorem 3.5 to find a \\
square with the maximum point-weight. \\
3. Return the square found in the previous step. \\
\hline
\end{tabular}

Theorem 3.6. Given a trajectory $T$ in $\mathbb{R}^{2}$ and the value of $s$, Algorithm 3.2 finds a $(1+\epsilon)$-approximate hotspot of trajectory $T$ with time complexity $O\left(\frac{n \phi}{\epsilon} \log \frac{n \phi}{\epsilon}\right)$, in which $\phi$ is the ratio of average length of trajectory edges to $s$.

Proof. After tiling in the first step of this algorithm, an edge of length $d$ is subdivided into at most $\left\lceil\frac{2 d}{\epsilon s}\right\rceil$ segments. Therefore, if the total length of the edges of $T$ is $a$,

$$
m \leq \frac{2 a}{\epsilon S}+n,
$$


in which $m$ is the number of resulting segments. Thus, $m$ is equal to $O\left(\frac{n \phi}{\epsilon}\right)$, asymptotically. As shown in Theorem 3.5, finding a square with the maximum point-weight, $r$, can be found in $O(m \log m)$, where $m$ is the number of points after tiling. Let $r^{\prime}$ be the square with the same centre of gravity as $r$ but of side length $s+\epsilon s$. Also, let $h$ denote the weight of an exact hotspot of $T$. We show that $r^{\prime}$ is a $(1+\epsilon)$-approximate hotspot.

Lemma 3.2 implies that there is at least one square with side length $s+\epsilon s / 2$ whose point-weight is equal to $h$, the weight of an exact hotspot of $T$ (of side length $s$ ). Since $r$ is the square with the maximum point-weight among squares of side length $s+\epsilon s / 2$, its point-weight is at least $h$. Furthermore, Lemma 3.3 shows that the weight of $r^{\prime}$ is at least the point-weight of $r$ (at least $h$ ). Therefore, the algorithm finds a square of side length $s+\epsilon s$ and with weight at least $h$, which is a $(1+\epsilon)$-approximate hotspot by Definition 2.4.

We now use the algorithm presented in Theorem 3.6 to find a duration-approximate hotspot of a trajectory in the plane. For that, we need Lemma 3.7.

Lemma 3.7. Let $h$ be the weight of an exact hotspot of a trajectory $T$ in the plane. There exists a square of side length $c s$ and weight at least $h / 4$, provided that $c \geq 1 / 2$.

Proof. Let $r$ be a square of weight $h$, and therefore an exact hotspot of $T$. Place four squares $r_{1}, r_{2}, r_{3}$ and $r_{4}$ of side length $c s$ inside $r$ such that each square contains a separate corner of $r$. Since $c \geq 1 / 2$, these squares contain the centre of $r$ and each part of $r$ is covered by at least one of these squares. This implies that

$$
w(r) \leq w\left(r_{1}\right)+w\left(r_{2}\right)+w\left(r_{3}\right)+w\left(r_{4}\right) .
$$

The weight of at least one of these smaller squares should be at least $w / 4$. Otherwise,

$$
w(r)<4 \cdot h / 4,
$$

which is a contradiction.

We can use Theorem 3.6 to obtain Theorem 3.8.

Theorem 3.8. Given a trajectory $T$ in $\mathbb{R}^{2}$ with $n$ edges and the value of $s$, there is an algorithm that finds a 1/4duration-approximate hotspot of $T$ with time complexity $O(n \phi \log n \phi)$, in which $\phi$ is the ratio of average length of trajectory edges to $s$.

Proof. Let $h_{s}$ and $h_{s / 2}$ be weights of a hotspot of side length $s$ and $s / 2$, respectively. Theorem 3.6 for the hotspot of side length $s^{\prime}=s / 2$ and $\epsilon=1$ yields a square $r$ of weight at least $h_{s / 2}$ and side length $s^{\prime}+\epsilon s^{\prime}=s$. Since $h$ is the maximum weight of the squares with side length $s / 2$, Lemma 3.7 implies that the weight of an exact hotspot of side length $s$ of $T$ cannot be greater than $4 h_{s / 2}$. We thus have

$$
w(r) \geq h_{s / 2} \geq h_{s} / 4 .
$$

Therefore, $r$ is a 1/4-duration-approximate hotspot of $T$.

\section{Conclusion}

We presented an algorithm to find a $(1+\epsilon)$-approximate hotspot of a trajectory with $n$ edges on the plane for $\epsilon>0$ with time complexity $O\left(\frac{n \phi}{\epsilon} \log \frac{n \phi}{\epsilon}\right)$, where $\phi$ is the ratio of average trajectory edge length to $s$. We used this algorithm to find 1/4-duration-approximate hotspots of a trajectory with time complexity $O(n \phi \log n \phi)$, and if $\phi$ is bounded by a constant, $O(n \log n)$.

Several related problems seem interesting for further investigation. The approximation ratio of Theorem 3.8 may be improved. Also, instead of processing the whole trajectory and finding its stay regions, it would be useful to find the weight of any query square after some preprocessing. Furthermore, it seems useful in some applications to find a hotspot of not the whole trajectory, but only a specific interval or based on the geographic context [11], which is given as a query.

\section{References}

[1] van Kreveld M.J., Löffler M., Wiratma L., On Optimal Polyline Simplification Using the Hausdorff and Fréchet Distance, in International Symposium on Computational Geometry, 2018, 56:156:14, 10.4230/LIPICS.SOCG.2018.56

[2] Aronov B., Driemel A., van Kreveld M.J., Löffler M., Staals F., Segmentation of Trajectories on Nonmonotone Criteria, ACM Transactions on Algorithms, 12(2), 2016, 26:1-26:28, $10.1145 / 2660772$

[3] Buchin K., Buchin M., van Kreveld M.J., Speckmann B., Staals F., Trajectory grouping structure, Journal of Computational Geometry, 6(1), 2015, 75-98, 10.20382/JOCG.V6I1A3

[4] Alewijnse S.P.A., Buchin K., Buchin M., Sijben S., Westenberg M.A., Model-Based Segmentation and Classification of Trajectories, Algorithmica, 80(8), 2018, 2422-2452, 10.1007/S00453017-0329-X

[5] Benkert M., Djordjevic B., Gudmundsson J., Wolle T., Finding Popular Places, International Journal of Computational Geometry \& Applications, 20(1), 2010, 19-42, 10.1142/S02181959100 03189

[6] Gudmundsson J., van Kreveld M.J., Staals F., Algorithms for Hotspot Computation on Trajectory Data, in International Con- 
ference on Advances in Geographic Information Systems, 2013, 134-143, 10.1145/2525314.2525359

[7] Damiani M.L., I. H., Cagnacci F., Extracting stay regions with uncertain boundaries from GPS trajectories: a case study in animal ecology, in ACM International Conference on Advances in Geographic Information Systems (SIGSPATIAL), 2014, 253-262, 10.1145/2666310.2666417

[8] Rudi A.G., Looking for Bird Nests: Identifying Stay Points with Bounded Gaps, in The Canadian Conference on Computational Geometry, 2018, 334-339
[9] Rudi A.G., Approximate Hotspots of Orthogonal Trajectories, Fundamenta Informaticae, 167(4), 2019, 271-285, 10.3233/FI2019-1818

[10] de Berg M., Cheong 0., van Kreveld M.J., Overmars M.H., Computational Geometry - Algorithms and Applications, Springer, third edition, 2008, 10.1007/978-3-540-77974-2

[11] Buchin M., Dodge S., Speckmann B., Similarity of trajectories taking into account geographic context, Journal of Spatial Information Science, 9(1), 2014, 101-124, 10.5311/JOSIS.2014.9.179 